\title{
CONTINUOUS DEPENDENCE RESULTS FOR A CLASS OF EVOLUTION INCLUSIONS
}

\author{
by NIKOLAOS S. PAPAGEORGIOU*
}

\begin{abstract}
In this paper we examine the dependence of the solutions of an evolution inclusion on a parameter $\lambda$. We prove two dependence theorems. In the first the parameter appears only in the orientor field and we show that the solution set depends continuously on it for both the Vietoris and Hausdorff topologies. In the second the parameter appears also in the monotone operator. Using the notion of $G$-convergence of operators we prove that the solution set is upper semicontinuous with respect to the parameter. Both results make use of a general existence theorem which we also prove in this paper. Finally, we present two examples. One from control theory and the other from partial differential inclusions.
\end{abstract}

1980 Mathematics subject classification (1985 Revision): 34G20

\section{Introduction}

In this paper we study the dependence of the solutions of a nonlinear evolution inclusion on a parameter. Previous work in this direction was done by Stassinopoulos and Vinter [11], Vasilev [14] and Lim [5] for differential inclusions in $\mathbb{R}^{n}$ and by Tolstonogov [13] and Papageorgiou [8] for differential inclusions in a Banach space. However, the systems considered by Tolstonogov [13] and Papageorgiou [8] did not allow for the presence of unbounded operators and therefore did not cover the important case of partial differential equations with multivalued terms. Very recently Frankowska [17] and Papageorgiou [18] studied evolution inclusions in Banach spaces. In both the above papers the systems considered were semilinear (i.e. the unbounded operator $A$ was linear and generated a compact semigroup; Frankowska [17] considered also alternative compactness hypotheses, see Corollary 2.6, p. 113). Also in both the above works the unbounded operator was time independent. For such systems the authors established relaxation results. Furthermore Frankowska [17] went on and obtained useful estimates and variational inclusions governing the reachable sets, while Papageorgiou [18] allowed the multivalued perturbation term $F$ to depend on a control variable $u$ and for the resulting control system studied Lagrange and time optimal control problems. In this paper we consider evolution inclusions with nonlinear and time dependent unbounded operators. So instead of relying on semigroup theory as in Frankowska [17] and Papageorgiou [18], we adopt the formalism of Lions [6] and Zeidler [19] which involves "Gelfand or evolution triples" of spaces (see Section 2). For

\footnotetext{
*Research supported by NSF Grant DMS-8802688.
} 
such systems we prove two continuous dependence results. In the first the parameter appears only in the multivalued term (orientor field). For this type of systems, under some appropriate continuity hypotheses, we prove that the solution set of the evolution inclusion depends on the parameter continuously for both the Vietoris and Hausdorff topologies. In the second result, we allow the parameter to appear in the operator which models the partial differential term. Using the notion of $G$-convergence, we establish the upper semicontinuity of the solution set on the parameter. Both continuous dependence results make use of a general existence result which we also prove in this paper. In the last section we present two examples, one from control theory and the other partial differential inclusions.

\section{Preliminaries}

Let $(\Omega, \Sigma)$ be a measurable space and $X$ a separable Banach space. We will be using the following notation:

$$
P_{f(c)}(X)=\{A \subseteq X: \text { nonempty, closed, (convex) }\}
$$

and

$$
P_{k(c)}(X)=\{A \subseteq X: \text { nonempty, compact, (convex) }\} .
$$

A multifunction $F: \Omega \rightarrow P_{f}(X)$ is said to be measurable, if for every $z \in X$ $\omega \rightarrow d(z, F(\omega))=\inf \{\|z-x\|: x \in F(\omega)\}$ is measurable. A multifunction $F: \Omega \rightarrow 2^{X} \backslash\{\varnothing\}$ is said to be graph measurable, if $\operatorname{Gr} F=\{(\omega, x) \in \Omega \times X: x \in F(\omega)\} \in \Sigma \times B(X)$, with $B(X)$ being the Borel $\sigma$-field of $X$. For $P_{f}(X)$-valued multifunctions, measurability implies graph measurability, while the converse is true if there is a $\sigma$-finite measure $\mu(\cdot)$ on $(\Omega, \Sigma)$, with respect to which $\Sigma$ is complete. Fo: more details we refer to the survey paper of Wagner [15]. By $S_{F}^{p}(1 \leqq p \leqq \infty)$ we will denote the set of selectors of $F(\cdot)$ that belong in the Lebesgue-Bochner space $L^{p}(X)$; i.e. $S_{F}^{p}=\left\{f \in L^{p}(X): f(\omega) \in F(\omega) \mu\right.$-a.e. $\}$. Note that for a graph measurable multifunction $F: \Omega \rightarrow 2^{X} \backslash\{\varnothing\}$, $S_{F}^{P}$ is nonempty if and only if $\omega \rightarrow \inf \{\|z\|: z \in F(\omega)\} \in L_{+}^{p}$. In particular this is the case if $\omega \rightarrow|F(\omega)|=\sup \{\|z\|$ $: z \in F(\omega)\} \in L_{+}^{p}$ and such a multifunction is usually called $L^{p}$-integrably bounded.

Let $Y, Z$ be Hausdorff topological spaces, A multifunction $G: Y \rightarrow 2^{Z} \backslash\{\varnothing\}$ is said to be upper semicontinuous (u.s.c.) (resp. lower semicontinuous (l.s.c.)) if for every $U \subseteq Z$ open $G^{+}(U)=\{y \in Y: G(y) \subseteq U\}$ (resp. $G^{-}(U)=\{y \in Y: G(y) \cap U \neq \varnothing\}$ ) is open in $Y$. If $Y, Z$ are metric spaces, then the above definition of lower semicontinuity is equivalent to saying that if $y_{n} \rightarrow y$, then $G(y) \subseteq \lim G\left(y_{n}\right)=\left\{z \in Z: \lim d\left(z, G\left(y_{n}\right)\right)=0\right\}=\left\{z \in Z: z=\lim z_{n}, z_{n} \in A_{n}\right.$, $n \geqq 1\}$. Also if we assume additional structure on $Y, Z$ and on the values of $G(\cdot)$, then we can also have other equivalent definitions of upper semicontinuity. For details we refer to Delahaye and Denel [3] and Klein and Thompson [4]. A multifunction $G: Y \rightarrow 2^{z} \backslash\{\varnothing\}$ is said to be continuous, if it is both u.s.c. and l.s.c. Note that this is continuity of $G(\cdot)$ from $Y$ into $2^{Z} \backslash\{\varnothing\}$ with the Vietoris topology.

Let $Z$ be a metric space. On $P_{f}(Z)$ we can define a (generalized) metric, known in the literature as Hausdorff metric, by setting $h(A, B)=\max \left\{\sup _{a \in A} d(a, B), \sup _{b \in B} d(b, A)\right\}$. If 
$Z$ is complete, then so is $\left(P_{f}(Z), h\right)$. A multifunction $G: Y \rightarrow P_{f}(Z)$ is Hausdorff continuous ( $h$-continuous), if it is continuous from the topological space $Y$ into the metric space $\left(P_{f}(Z), h\right)$. In general continuity and $h$-continuity are disjoint notions but they coincide if $G(\cdot)$ is $P_{k}(Z)$-valued. If $\left\{A_{n}\right\}_{n \geqq 1} \subseteq 2^{Z} \backslash\{\varnothing\}$, then we define $\overline{\lim } A_{n}=\left\{z \in Z: \underline{\lim } d\left(z, A_{n}\right)=0\right\}=\left\{z \in Z: z=\lim z_{n_{k}}, z_{n_{k}} \in A_{n_{k}}, n_{1}<n_{2}<\cdots<n_{k}<\cdots\right\}$. When $Z$ is a Banach space, we can also define $w$ - $\overline{\lim } A_{n}=\{z \in Z: z=$ $\left.w-\lim z_{n_{k}}, z_{n_{k}} \in A_{n_{k}}, n_{1}<\cdots<n_{k}<\cdots\right\}$, where $w$ - denotes the weak topology on $Z$. Note that $\overline{\lim } A_{n} \subseteq w-\overline{\lim } A_{n}$.

Now let $H$ be a separable Hilbert space and let $X$ be a dense subspace of $H$ carrying the structure of a separable Hilbert space s.t. $X \hookrightarrow H$ compactly. Then identifying $H$ with its dual (pivot space), we have $X \hookrightarrow H \hookrightarrow X^{*}$ with all embeddings being compact. To have a concrete example in mind let $H=L^{2}(0,1), X=H_{0}^{m}(0,1)$ and $X^{*}=H^{-m}(0,1)$. The classical Sobolev-Kondrachov embedding theorem tells us that $X \hookrightarrow H \hookrightarrow X^{*}$ compactly. Such a triple of spaces is known in the literatures as a "Gelfand triple". By $\|\cdot\|$ (resp. $|\cdot|,\|\cdot\|_{*}$ ) we will denote the norm of $X$ (resp. of $H, X^{*}$ ). Also by $(\cdot, \cdot)$ we will denote the inner produce of $H$ and by $\langle\cdot, \cdot\rangle$ the duality brackets for the pair $\left(X, X^{*}\right)$. The two are compatible in the sense that $\left.\langle\cdot, \cdot\rangle\right|_{X \times H}=(\cdot, \cdot)$. Let $W(T)=$ $\left\{x(\cdot) \in L^{2}(X): \dot{x} \in L^{2}\left(X^{*}\right)\right\}$ (the derivative here being the distributional derivative). Furnished with the inner product $(x, y)_{W(T)}=\int_{0}^{b}(x(t), y(t))_{X} d t+\int_{0}^{b}(\dot{x}(t), \dot{y}(t))_{X^{*}} d t, W(T)$ becomes a Hilbert space. Furthermore, it is well known that $W(T) \hookrightarrow C(T, H)$, i.e. every element of $W(T)$ after possible modification on a Lebesgue null set is equal to an $H$ valued continuous function. In addition, from Nagy [7], we know that the above embedding is compact.

Let $\left(X, H, X^{*}\right)$ be a Gelfand triple as above and let $\left\{A_{n}, A\right\}_{n \geqq 1} \subseteq \mathscr{L}\left(X, X^{*}\right)$. Following Zhikov, Kozlov and Oleinik [16], we make the following two definitions.

Definition 1. We say that the sequence $\left\{A_{n}\right\}_{n \geqq 1} G$-converges to $A$ if and only if for every $n \geqq 1, A_{n}^{-1}, A^{-1} \in \mathscr{L}\left(X^{*}, X\right)$ exist and for every $x^{*} \in X^{*} A_{n}^{-1} x^{*} \stackrel{w}{\rightarrow} A^{-1} x^{*}$ in $X$ (and hence also strongly in $H$ ) as $n \rightarrow \infty$.

Definition 2. We say that a sequence of operators $P_{n}: W(T) \rightarrow L^{2}\left(X^{*}\right) \times H \quad P G$ converges to an operator $P: W(T) \rightarrow L^{2}\left(X^{*}\right) \times H$ as $n \rightarrow \infty$, if for all $n \geqq 1$ the operators $P_{n}^{-1}, P^{-1}: L^{2}\left(X^{*}\right) \times H \rightarrow W(T)$ are defined and for any $\left(f, x_{0}\right) \in L^{2}\left(X^{*}\right) \times$ $H, P_{n}^{-1}\left(f, x_{0}\right) \stackrel{w}{\rightarrow-1}\left(f, x_{0}\right)$ in $W(T)$ (which implies that for all $t \in T$, $P_{n}^{-1}\left(f, x_{0}\right)(t) \stackrel{s}{\rightarrow} P^{-1}\left(f, x_{0}\right)(t)$ in $\left.H\right)$.

In our case for every $n \geqq 1 \quad P_{n}: x \in W(T) \rightarrow\left(\dot{x}(\cdot)+A_{n}(\cdot) x(\cdot), x(0)\right) \in L^{2}\left(X^{*}\right) \times H$. So $G-$ convergence refers to the convergence of the solutions of a sequence of elliptic problems, while $P G$-convergence refers to the convergence of the solutions of a sequence of parabolic problems.

It must be noted that the notion of $G$-convergence of parabolic operators was first introduced by Spagnolo [20], who later, in [21], obtained an important compactness result for this mode of convergence. In [22] Colombini and Spagnolo obtained useful conditions on the coefficients guaranteeing $P G$-convergence of the corresponding 
parabolic operators. The abstract theory of $G$ and $P G$-convergence was developed in Zhikov, Kozlov and Oleinik [16].

Finally if $V$ is a Banach space and $A \in 2^{V} \backslash\{\varnothing\}$, then by $\sigma_{A}: V^{*} \rightarrow \mathbb{R}$ we denote the support function of $A$, i.e. $\sigma_{A}\left(v^{*}\right)=\sup _{a \in A}\left(v^{*}, a\right)$.

\section{Continuous dependence results}

We will start with a general existence theorem.

So let $T=[0, r]$ and $\left(X, H, X^{*}\right)$ a Gelfand triple of spaces as in Section 2. We consider the following multivalued Cauchy problem on $T$ :

$$
\left\{\begin{array}{l}
\dot{x}(t)+A(t, x(t)) \in F(t, x(t)) \text { a.e. } \\
x(0)=x_{0}
\end{array}\right\}
$$

Here $A: T \times X \rightarrow X^{*}$ and $F: T \times H \rightarrow P_{f c}(H)$. By a solution of $\left(^{*}\right)$ we understand a map $x(\cdot) \in W(T) \hookrightarrow C(T, H)$ s.t. for some $f(\cdot) \in S_{F(\cdot, x(\cdot))}^{2}$ we have that $\dot{x}(t)+A(t, x(t))=f(t)$ a.e., $x(0)=x_{0}$.

We will need the following hypotheses on the data of $\left(^{*}\right)$.

$H(A): \quad A: T \times X \rightarrow X^{*}$ is an operator s.t.

(1) $t \rightarrow A(t, x)$ is measurable,

(2) $x \rightarrow A(t, x)$ is hemicontinuous, monotone,

(3) $\|A(t, x)\|_{*} \leqq a(1+\|x\|)$ a.e. with $a>0$,

(4) $\langle A(t, x), x\rangle \geqq c\|x\|^{2}$ a.e. with $c>0$.

$H(F): \quad F: T \times H \rightarrow P_{f c}(H)$ is a multifunction s.t.

(1) $F(\cdot, \cdot)$ is graph measurable,

(2) $F(t, \cdot)$ has a sequentially closed graph in $H \times H_{w}$, where $H_{w}$ denotes the Hilbert space $H$ with the weak topology,

(3) $|F(t, x)| \leqq a(t)+b|x|$ a.e. with $a(\cdot) \in L_{+}^{2}, b>0$.

Theorem 3.1. If hypotheses $H(A), H(F)$ hold and $x_{0} \in H$ then $\left({ }^{*}\right)$ admits a solution.

Proof. First we will derive some a priori bounds for the solutions of (*). So let $x(\cdot) \in W(T)$ be such a solution. By definition we have

$$
\left\{\begin{array}{l}
\dot{x}(t)+A(t, x(t))=h(t) \text { a.e. } \\
x(0)=x_{0}
\end{array}\right\}
$$

with $h(\cdot) \in S_{F(\cdot, x(\cdot))}^{2}$. Multiply the above evolution equation with $x(\cdot)$. We get: 


$$
\begin{aligned}
&\langle\dot{x}(t), x(t)\rangle+\langle A(t, x(t)), x(t)\rangle=(h(t), x(t)) \text { a.e. } \\
& \Rightarrow \frac{d}{d t}|x(t)|^{2}+2 c\|x(t)\|^{2} \leqq 2|h(t)| \cdot|x(t)| \text { a.e. } \\
& \Rightarrow|x(t)|^{2}+2 c \int_{0}^{t}\|x(s)\|^{2} d s \leqq \leqq\left.\int_{0}^{t}|h(s)||\cdot| x(s)|d s+| x_{0}\right|^{2} \\
& \leqq \int_{0}^{t}|h(s)|^{2} d s+\int_{0}^{t}|x(s)|^{2} d s+\left|x_{0}\right|^{2} \\
& \leqq \int_{0}^{t}\left(2 a(s)^{2}+2 b^{2}|x(s)|^{2}\right) d s+\int_{0}^{t}|x(s)|^{2} d s+\left|x_{0}\right|^{2} \\
&=\int_{0}^{t}\left(2 a(s)^{2}+\left(2 b^{2}+1\right)|x(s)|^{2}\right) d s+\left|x_{0}\right|^{2} \\
& \Rightarrow \quad|x(t)|^{2} \leqq \int_{0}^{t}\left(2 a(s)^{2}+\left(2 b^{2}+1\right)|x(s)|^{2}\right) d s+\left|x_{0}\right|^{2} \\
& \Rightarrow|x(t)| \leqq M \text { for some } M>0 \text { and for all } t \in T(\text { Gronwall's inequality). }
\end{aligned}
$$

Then we have:

$$
\begin{gathered}
2 c \int_{0}^{t}\|x(s)\|^{2} d s \leqq \int_{0}^{t}\left(a(s)^{2}+\left(2 b^{2}+1\right) M^{2}\right) d s+\left|x_{0}\right|^{2}, \quad t \in T \\
\Rightarrow\|x(\cdot)\|_{L^{2}(X)} \leqq M_{1} \text { for some } M_{1}>0
\end{gathered}
$$

Finally let $p(\cdot) \in L^{2}(X)$. We have

$$
\begin{aligned}
& \int_{0}^{t}\langle\dot{x}(s), p(s)\rangle d s \leqq \int_{0}^{t}\|A(s, x(s))\|_{*} \cdot\|p(s)\| d s+\int_{0}^{t}\|h(s)\|_{*}\|p(s)\| d s \\
& \leqq \int_{0}^{t}(c(1+\|x(s)\|)+a(s)+b|x(s)|)\|p(s)\| d s \\
& \leqq\left(\|c+a(\cdot)+b M\|_{2}+c\|x(\cdot)\|_{L^{2}(X)}\right) \cdot\|p(\cdot)\|_{L^{2}(X)} \\
& \Rightarrow \quad((\dot{x}, p))_{0} \leqq\left(\|c+a(\cdot)+b M\|_{2}+c M_{1}\right) \cdot\|p\|_{L^{2}(X)}
\end{aligned}
$$

where $((\cdot, \cdot))_{0}$ denotes the duality brackets for the pair $\left(L^{2}(X), L^{2}\left(X^{*}\right)\right)$. Hence

$$
\|\dot{x}\|_{L^{2}\left(X^{*}\right)} \leqq\|c+a(\cdot)+b M\|_{2}+c M_{1}=M_{2} .
$$

Define $\hat{F}: T \times H \rightarrow P_{f c}(H)$ by 


$$
\hat{F}(t, x)= \begin{cases}F(t, x) & \text { if }|x| \leqq M \\ F\left(t, \frac{M x}{|x|}\right) & \text { if } \quad|x|>M\end{cases}
$$

Observe that $\hat{F}(t, x)=F\left(t, p_{M}(x)\right)$, where $p_{M}$ is the $M$-radial retraction. Recalling that $p_{M}(\cdot)$ is Lipschitz continuous, it is easy to check that $\hat{F}(\cdot, \cdot)$ has the same measurability and continuity properties as $F(\cdot, \cdot)$ (i.e. it satisfies hypotheses $H(F)(1)$ and (2)) and furthermore $|\hat{F}(t, x)| \leqq a(t)+b M=\hat{a}(t)$ a.e. with $\hat{a}(\cdot) \in L_{+}^{2}$.

Now set $V=\left\{h \in L^{2}(H):|h(t)| \leqq \hat{a}(t)\right.$ a.e. $\}$ and define $p: V \rightarrow W(T)$ to be the map that to each $h \in V$, assigns the unique solution of the evolution equation

$$
\left\{\begin{array}{l}
\dot{x}(t)+A(t, x(t))=h(t) \text { a.e. } \\
x(0)=x_{0}
\end{array}\right\}
$$

(see Theorem 4.2, p. 167 of Barbu [1] and also Theorem 1.2, p. 162 of Lions [6]).

Our claim is that $p(\cdot)$ is sequentially weakly continuous. To this end let $h_{n} \stackrel{\omega}{\rightarrow} h$ in $V \subseteq L^{2}(H)$. Set $x_{n}=p\left(h_{n}\right) \in W(T)$. From our a priori estimation in the beginning of the proof, we know that $\left\{x_{n}(\cdot)\right\}_{n \geqq 1}$ is bounded in $W(T)$, hence relatively sequentially weakly compact in that space. Thus by passing to a subsequence if necessary, we may assume that $x_{n} \stackrel{w}{\rightarrow} x$ in $W(T)$. We have:

$$
\dot{x}_{n}(t)+A\left(t, x_{n}(t)\right)=h_{n}(t) \text { a.e. }
$$

Multiply the above equation with $x_{n}(t)-x(t)$. We get

$$
\begin{gathered}
\left\langle\dot{x}_{n}(t), x_{n}(t)-x(t)\right\rangle+\left\langle A\left(t, x_{n}(t)\right), x_{n}(t)-x(t)\right\rangle=\left(h_{n}(t), x_{n}(t)-x(t)\right) \text { a.e. } \\
\Rightarrow \int_{0}^{r}\left\langle\dot{x}_{n}(t), x_{n}(t)-x(t)\right\rangle d t+\int_{0}^{r}\left\langle A\left(t, x_{n}(t)\right), x_{n}(t)-x(t)\right\rangle d t=\int_{0}^{r}\left(h_{n}(t), x_{n}(t)-x(t)\right) d t .
\end{gathered}
$$

From Lemma 5.5.1, p. 151 of Tanabe [12], we have

$$
\begin{gathered}
\left\langle\dot{x}_{n}(t)-\dot{x}(t), x_{n}(t)-x(t)\right\rangle=\frac{1}{2} \frac{d}{d t}\left|x_{n}(t)-x(t)\right|^{2} \text { a.e. } \\
\Rightarrow \int_{0}^{r}\left\langle\dot{x}_{n}(t)-\dot{x}(t), x_{n}(t)-x(t)\right\rangle d t=\frac{1}{2}\left|x_{n}(r)-x(r)\right|^{2} \\
\Rightarrow \int_{0}^{r}\left\langle\dot{x}_{n}(t), x_{n}(t)-x(t)\right\rangle d t=\frac{1}{2}\left|x_{n}(r)-x(r)\right|^{2}+\int_{0}^{r}\left\langle\dot{x}(t), x_{n}(t)-x(t)\right\rangle d t .
\end{gathered}
$$


So we get

$$
\begin{aligned}
& \frac{1}{2}\left|x_{n}(r)-x(r)\right|^{2}+\int_{0}^{r}\left\langle\dot{x}(t), x_{n}(t)-x(t)\right\rangle d t+\int_{0}^{r}\left\langle A\left(t, x_{n}(t)\right), x_{n}(t)-x(t)\right\rangle d t \\
& \quad=\int_{0}^{r}\left(h_{n}(t), \dot{x}_{n}(t)-x(t)\right) d t .
\end{aligned}
$$

Since $W(T) \hookrightarrow C(T, H)$ compactly (see Section 2), we may also assume that $x_{n} \stackrel{s}{\rightarrow} x$ in $C(T, H)$. So we have

$$
\begin{gathered}
\frac{1}{2}\left|x_{n}(r)-x(r)\right|^{2} \rightarrow 0 \\
\int_{0}^{r}\left\langle\dot{x}(t), x_{n}(t)-x(t)\right\rangle d t \rightarrow 0
\end{gathered}
$$

and

$$
\int_{0}^{r}\left(h_{n}(t), x_{n}(t)-x(t)\right) d t \rightarrow 0
$$

Therefore if $\hat{A}: L^{2}(X) \rightarrow L^{2}\left(X^{*}\right)$ is the Nemitsky operator corresponding to $A(t, x)$ (i.e. $(\hat{A} x)(t)=A(t, x(t)))$, we have

$$
\lim \left(\left(\hat{A} x_{n}, x_{n}-x\right)\right)_{0}=0 .
$$

Note that $\left\{\hat{A} x_{n}\right\}_{n \geqq 1}$ is bounded in $L^{2}\left(X^{*}\right)$ (hypothesis $H(A)(3)$ ) and so by passing to a subsequence if necessary, we may assume that $\hat{A} x_{n} \stackrel{w}{\rightarrow} w$ in $L^{2}\left(X^{*}\right)$. Furthermore, it is easy to see that $\hat{A}(\cdot)$ is hemicontinuous and monotone. Hence invoking Proposition 2.5, p. 179 of Lions [6], we get that $w=\hat{A} x$, i.e. $\hat{A} x_{n} \stackrel{w}{\rightarrow} \hat{A} x$ in $L^{2}\left(X^{*}\right)$.

Then for every $p(\cdot) \in L^{2}(X)$, we have

$$
\begin{gathered}
\left(\left(\dot{x}_{n}, p\right)\right)_{0}+\left(\left(\hat{A} x_{n}, p\right)\right)_{0}=\left(\left(h_{n}, p\right)\right)_{0} \\
\rightarrow((\dot{x}, p))_{0}+((\hat{A} x, p))_{0}=((h, p))_{0} \quad \text { as } n \rightarrow \infty . \\
\Rightarrow \quad \dot{x}(t)+A(t, x(t))=h(t) \quad \text { a.e., } x(0)=x_{0} .
\end{gathered}
$$

Also from Theorem 3.1 of Papageorgious [9], we have

$$
h(t) \in \overline{\operatorname{conv}} w-\varlimsup \overline{\lim }\left\{h_{n}(t)\right\}_{n \geqq 1} \subseteq \overline{\operatorname{conv}} w-\varlimsup \overline{\lim } \hat{F}\left(t, x_{n}(t)\right)
$$

for all $t \in T \backslash N \mu(N)=0$ (here $\mu(\cdot)$ denotes the Lebesgue measure on $T$ ). Let $t \in T \backslash N$ and 
$v \in w-\varlimsup i m\left(t, x_{n}(t)\right)$. Then by definition (see Section 2) we can find $\left\{x_{n_{k}}: k \geqq 1, n_{1}<n_{2}<\right.$ $\left.\cdots<n_{k}<\cdots\right\}$ and $v_{n_{k}} \in \hat{F}\left(t, x_{n_{k}}(t)\right)$ s.t. $v_{n_{k}} \stackrel{w}{\rightarrow} v$ in $H$. Note that $\left(x_{n_{k}}(t), v_{n_{k}}\right) \in \operatorname{Gr} \hat{F}(t, \cdot)$ and the latter is sequentially weakly closed in $H \times H_{w}$. So $(x(t), v) \in \operatorname{Gr} \hat{F}(t, \cdot) \Rightarrow v \in \hat{F}(t, x(t)) \Rightarrow$ $w-\overline{\lim } \hat{F}\left(t, x_{n}(t)\right) \subseteq F(t, x(t))$ for all $t \in T \backslash N, \mu(N)=0, \Rightarrow \overline{\operatorname{conv}} w-\varlimsup \overline{\lim } \hat{F}\left(t, x_{n}(t)\right) \subseteq \hat{F}(t, x(t))$

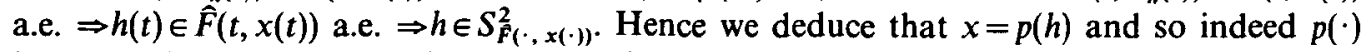
is sequentially weakly continuous as claimed.

Next let $R: V \rightarrow P_{f c}(V)$ be defined by

$$
R(h)=S_{\hat{F}(\cdot, p(h)(\cdot))}^{1}
$$

Our claim is that $R(\cdot)$ is u.s.c. on $V$ endowed with the relative weak $L^{2}(H)$-topology (denoted henceforth by $V_{w}$ ). Note that $V_{w}$ is compact metrizable. So in order to show that $R(\cdot)$ is u.s.c., it is enough to show that $\operatorname{Gr} R$ is sequentially closed in $V_{w} \times V_{w}$. So let $\left\{\left(h_{n}, f_{n}\right)\right\}_{n \geqq 1} \subseteq \mathrm{Gr} R$ and assume that $\left(h_{n}, f_{n}\right) \stackrel{w \times w}{\longrightarrow}(h, f)$ in $V_{w} \times V_{w}$. Then we know that $p\left(h_{n}\right)(\cdot) \stackrel{w}{\rightarrow} p(h)(\cdot)$ in $W(T) \Rightarrow p\left(h_{n}\right)(t) \stackrel{s}{\rightarrow} p(h)(t)$ in $H$ for all $t \in T$. Using Theorem 4.2 of [9], we get

$$
\begin{gathered}
w-\varlimsup \lim R\left(h_{n}\right) \subseteq S_{\hat{F}(\cdot, p(h)(\cdot))}^{1}=R(h) \text { in } L^{2}(H) \\
\Rightarrow \quad(h, f) \in \mathrm{Gr} R \\
\Rightarrow \quad R(\cdot) \text { is u.s.c. as claimed. }
\end{gathered}
$$

Apply the Katutani-KyFan fixed point theorem to get $h \in V$ s.t. $h \in R(h)$. Then $x=p(h) \in W(T)$ solves $\left(^{*}\right)$ with the orientor field $\hat{F}(t, x)$. But as in the beginning of the proof, we can get that $|x(t)| \leqq M \Rightarrow \hat{F}(t, x(t))=F(t, x(t)) t \in T \Rightarrow x(\cdot)$ solves (*).

Remark. It is clear from the above proof that the solution set $S$ of $\left(^{*}\right)$ belongs in $P_{k}(C(T, H)$ ) (recall that $W(T) \hookrightarrow C(T, H)$ compactly; see Section 2).

In the proof of our first continuous dependence result we will need the following two lemmata.

Lemma $\alpha:$ If $F: T \times H \rightarrow P_{f c}(H)$ is a multifunction s.t. $t \rightarrow F(t, x)$ is measurable, $x \rightarrow F(t, x)$ is h-continuous and $v: T \rightarrow H$ is measurable then $(t, x) \rightarrow u(t, x)=\operatorname{proj}(v(t) ; F(t, x))$ is a Caratheodory map; i.e. measurable in $t$, continuous in $x$ (here proj $(\cdot, \cdot, F(t, x))$ is the metric projection on $F(t, x)$ ).

Proof. Note that $\operatorname{Gr} u(\cdot, x)=\{(t, z) \in T \times H: d(v(t), F(t, x))=|v(t)-z|\}$. Let $\eta: T \times H \rightarrow \mathbb{P}$ be defined by $\eta(t, z)=d(v(t), F(t, x))-|v(t)-z|$. Then $\operatorname{Gr} u(\cdot, x)=\{(t, z) \in T \times H: \eta(t, z)=0\}$. Observe that $\eta(\cdot, \cdot)$ is a Caratheodory function, hence jointly measurable. Thus Gr $u(\cdot, x)=\{(t, z) \in T \times H: \eta(t, z)=0\} \in B(T) \times B(H) \Rightarrow t \rightarrow u(t, x)$ is measurable.

Next let $x_{n} \rightarrow x$ in $H \Rightarrow F\left(t, x_{n}\right) \stackrel{h}{\rightarrow} F(t, x) \Rightarrow F\left(t, x_{n}\right) \stackrel{K \cdot M}{\longrightarrow} F(t, x) \Rightarrow u(t, \cdot)$ is continuous. 
Lemma $\beta$. If $Z$ is a metric space, $V$ is a Banach space, $F: Z \rightarrow P_{k}(V)$ is a multifunction s.t. for every $K \subseteq V$ nonempty, compact we have that $\left.F\right|_{K}$ is u.s.c., then $F(\cdot)$ is u.s.c.

Proof. We will show that for every $C \subseteq V$ nonempty, closed, $F^{-}(C)=\{z \in Z: F(z) \cap$ $C \neq \varnothing\}$ is closed in $Z$. So let $\left\{z_{n}\right\}_{n \geqq 1} \subseteq F^{-}(C)$ and assume $z_{n} \rightarrow z$. Set $K=\left\{z_{n}, z\right\}_{n \geqq 1}$. Then $K$ is compact and by hypothesis $\left.F\right|_{K}(\cdot)$ is u.s.c. Also since by hypothesis $F(\cdot)$ is $P_{k}(V)$-valued, from Theorem 7.4.2 of Klein and Thompson [4], we have that $F(K) \in P_{k}(V)$. Let $y_{n} \in F\left(z_{n}\right) \cap C, n \geqq 1$. Then $\left\{y_{n}\right\}_{n \geqq 1} \subseteq F(K)$ and so by passing to a subsequence if necessary we may assume that $y_{n} \rightarrow y \in C$. Also, since $\left.F\right|_{K}$ is u.s.c. we have $\varlimsup \lim F\left(z_{n}\right) \subseteq F(z)$ (see Delahaye and Denel [3]) $\Rightarrow y \in F(z) \Rightarrow y \in F(z) \cap C \Rightarrow z \in F^{-}(C) \Rightarrow F^{-}(C)$ is closed $\Rightarrow F(\cdot)$ is u.s.c.

Now we are ready for our first continuous dependence result. Assume $\Lambda$ is a metric space (the parameter space).

The multivalued Cauchy problem under consideration is the following:

$$
\left\{\begin{array}{l}
\dot{x}(t)+A(t, x(t)) \in F(t, x(t), \lambda) \text { a.e. } \\
x(0)=x_{0}(\lambda)
\end{array}\right\} .
$$

We will denote the solution set $\left({ }^{*}\right)_{\lambda}$ by $S(\lambda)$. From Theorem 3.1 (see also the remark following it), we know that $S(\lambda) \in P_{k}(C(T, H))$ for all $\lambda \in \Lambda$.

We will need the following hypotheses on the parametrized orientor field $F(t, x, \lambda)$ and the parametrized initial data:

$H(F)_{1}: F: T \times H \times \Lambda \rightarrow P_{f c}(H)$ is a multifunction s.t.

(1) $t \rightarrow F(t, x, \lambda)$ is measurable,

(2) $x \rightarrow F(t, x, \lambda)$ is $h$-Lipschitz with constant $k_{\lambda}(\cdot) \in L_{+}^{1}$ and $k_{B}(\cdot)=\sup _{\lambda \in B} k_{\lambda}(\cdot) \in L_{+}^{1}$ for every $B \subseteq \Lambda$ compact,

(3) $\lambda \rightarrow F(t, x, \lambda)$ is continuous,

(4) $|F(t, x, \lambda)| \leqq a_{\lambda}(t)+b_{\lambda}|x|$ a.e. with $a_{\lambda}(\cdot) \in L_{+}^{2}, b_{\lambda}>0$ and $a_{B}(\cdot)=\sup _{\lambda \in B} a_{\lambda}(\cdot) \in L_{+}^{2} b_{B}=$ $\sup _{\lambda \in B} b_{\lambda}<\infty$ for every $B \subseteq \Lambda$ compact.

$H_{0}: x_{0}: \Lambda \rightarrow H$ is continuous.

Theorem 3.2. If hypotheses $H(A), H(F)_{1}$ and $H_{0}$ hold, then $S: \Lambda \rightarrow P_{k}(C(T, H))$ is continuous and h-continuous.

Proof. First we will show that $S(\cdot)$ is 1.s.c. From Section 2, we know that it is enough to show that for $\lambda_{n} \rightarrow \lambda$ in $\Lambda$, we have $S(\lambda) \subseteq \underline{\lim } S\left(\lambda_{n}\right)$.

So let $x(\cdot) \in S(\lambda)$. We have

$$
\dot{x}(t)+A(t, x(t))=f(t) \text { a.e., } x(0)=x_{0}=x_{0} \text { with } f \in S_{F(\cdot, x(\cdot), \lambda)}^{2} .
$$

Define $v(t, \lambda)=\operatorname{proj}\left(f(t) ; F\left(t, x(t), \lambda_{n}\right)\right)$ and $u\left(t, z, \lambda_{n}\right)=\operatorname{proj}\left(v\left(t, \lambda_{n}\right) ; F\left(t, z, \lambda_{n}\right)\right)$. Observe 
that $u\left(t, x(t), \lambda_{n}\right)=v\left(t, \lambda_{n}\right)$. Also from Lemma $\alpha$, we know that $t \rightarrow u\left(t, z, \lambda_{n}\right)$ is measurable and $z \rightarrow u\left(t, z, \lambda_{n}\right)$ is continuous.

For every $n \geqq 1$, consider the following evolution equation:

$$
\left\{\begin{array}{l}
\dot{x}_{n}(t)+A\left(t, x_{n}(t)\right)=u\left(t, x_{n}(t), \lambda_{n}\right) \text { a.e. } \\
x_{n}(0)=x_{0}\left(\lambda_{n}\right)
\end{array}\right\}
$$

From Theorem 3.1 we know that the above Cauchy problem has at least one solution $x_{n}(\cdot) \in W(T)$. We have:

$$
\begin{gathered}
\left\langle\dot{x}(t)-\dot{x}_{n}(t), x(t)-x_{n}(t)\right\rangle+\left\langle A(t, x(t))-A\left(t, x_{n}(t)\right), x(t)-x_{n}(t)\right\rangle \\
=\left(f(t)-u\left(t, x_{n}(t), \lambda_{n}\right), x(t)-x_{n}(t)\right) \text { a.e. } \\
\Rightarrow\left|x(t)-x_{n}(t)\right|^{2} \leqq \int_{0}^{t}\left(f(s)-u\left(s, x_{n}(s), \lambda_{n}\right), x(s)-x_{n}(s)\right) d s+\left|x_{0}-x_{0}\left(\lambda_{n}\right)\right|^{2} \\
\leqq \int_{0}^{t}\left|f(s)-u\left(s, x_{n}(s), \lambda_{n}\right)\right| \cdot\left|x(s)-x_{n}(s)\right| d s+\left|x_{0}-x_{0}\left(\lambda_{n}\right)\right|^{2} \\
\leqq \int_{0}^{t}\left(\left|f(s)-u\left(s, x(s), \lambda_{n}\right)\right|+\left|u\left(s, x(s), \lambda_{n}\right)-u\left(s, x_{n}(s), \lambda_{n}\right)\right|\right) \cdot\left|x(s)-x_{n}(s)\right| d s+\left|x_{0}-x_{0}\left(\lambda_{n}\right)\right|^{2} \\
=\int_{0}^{t}\left(d\left(f(s), F\left(s, x(s), \lambda_{n}\right)\right)+d\left(v\left(s, \lambda_{n}\right), F\left(s, x_{n}(s), \lambda_{n}\right)\right)\right) \cdot\left|x(s)-x_{n}(s)\right| d s+\left|x_{0}-x_{0}\left(\lambda_{n}\right)\right|^{2} \\
\leqq \int_{0}^{t}\left(h\left(F(s, x(s), \lambda), F\left(s, x(s), \lambda_{n}\right)\right)+h\left(F\left(s, x(s), \lambda_{n}\right) F\left(s, x_{n}(s), \lambda_{n}\right)\right) \cdot\left|x(s)-x_{n}(s)\right| d s\right. \\
\quad+\left|x_{0}-x_{0}\left(\lambda_{n}\right)\right|^{2} .
\end{gathered}
$$

From the proof of Theorem 3.1 we know that for every $n \geqq 1\left|x_{n}(t)\right| \leqq M\left(\lambda_{n}\right) t \in T$ and for $B=\left\{\lambda_{n}, \lambda\right\}_{n \geqq 1} M_{B}=\sup _{\lambda \in B} M(\lambda)<\infty$ (see the proof of Theorem 3.1 and hypothesis $\left.H(F)_{1}\right)$. Also because of hypothesis $H(F)(3)$, we have $h\left(F(s, x(s), \lambda), F\left(s, x(s), \lambda_{n}\right)\right) \rightarrow 0$ as $n \rightarrow \infty$. So from the dominated convergence theorem, given $\varepsilon>0$, for $n \geqq 1$ large enough we will have

$$
\left.\int_{0}^{t} h\left(F(s, x(s), \lambda), F\left(s, x(s), \lambda_{n}\right)\right) \cdot\left|x(s)-x_{n}(s)\right| d s \leqq \int_{0}^{t} 2 M_{B} h(s, x(s), \lambda), F\left(s, x(s), \lambda_{n}\right)\right) d s<\varepsilon .
$$

Also from hypothesis $H(F)(2)$ we have that

$$
\int_{0}^{t} h\left(F\left(s, x(s), \lambda_{n}\right), F\left(s, x_{n}(s), \lambda_{n}\right)\right) \cdot\left|x(s)-x_{n}(s)\right| d s \leqq \int_{0}^{t} k_{B}(s) \cdot\left|x(s)-x_{n}(s)\right|^{2} d s
$$


So finally, for $n \geqq 1$ large enough, we have

$$
\left|x(t)-x_{n}(t)\right|^{2} \leqq \varepsilon+\int_{0}^{t} k_{B}(s) \cdot\left|x(s)-x_{n}(s)\right|^{2} d s .
$$

Invoking Gronwall's inequality, we get

$$
\left|x(t)-x_{n}(t)\right|^{2} \leqq \varepsilon \exp \left\|k_{B}\right\|_{1} \quad \text { for all } t \in T, \quad \Rightarrow \quad x_{n} \stackrel{s}{\rightarrow} x \text { in } C(T, H) .
$$

Clearly $x_{n}(\cdot) \in S\left(\lambda_{n}\right)$. So we have shown that

$$
S(\lambda) \subseteq \underline{\lim } S\left(\lambda_{n}\right) \quad \Rightarrow S(\cdot) \text { is l.s.c. }
$$

Next we will show that $S(\cdot)$ is u.s.c. From Lemma $\beta$, we know that it is enough to show that if $\lambda_{n} \rightarrow \lambda$ in $\Lambda$, then

$$
\varlimsup \lim S\left(\lambda_{n}\right) \subseteq S(\lambda)
$$

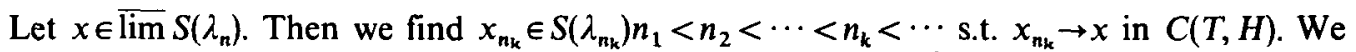
have $\dot{x}_{n_{k}}(t)+A\left(t, x_{n_{k}}(t)\right)=f_{n_{k}}(t)$ a.e., $x_{n_{k}}(0)=x_{0}\left(\lambda_{n_{k}}\right)$ with $\left.f_{n_{k}} \in S_{F(}^{2}, x_{n_{k}}(\cdot), \lambda_{n_{k}}\right)$. From the proof of Theorem 3.1 and hypothesis $H(F)_{1}$ we know that $\left\|x_{n_{k}}\right\|_{L^{2}(X)}^{\left(x^{2}\right.} \leqq M_{1 B^{\prime}},\left\|\dot{x}_{n_{k}}\right\|_{L^{2}\left(X^{*}\right)}$ $\leqq M_{2 B^{\prime}}$, where $B^{\prime}=\left\{\lambda_{n_{k}}, \lambda\right\}, M_{1 B^{\prime}}=\sup _{\lambda \in B^{\prime}} M_{1}(\lambda)<\infty$ and $M_{2 B^{\prime}}=\sup _{\lambda \in B^{\prime}} M_{2}(\lambda)<\infty$. So $\left\{x_{n_{k}}\right\}_{k \geqq 1} \subseteq W(T)$ is relatively sequentially weakly compact and thus by passing to a subsequence if necessary we may assume that $x_{n_{k}} \stackrel{w}{\rightarrow} x$ in $W(T)$. Also we know (see the proof of Theorem 3.1), that $\hat{A} x_{n_{k}} \stackrel{w}{\rightarrow} \hat{A} x$ in $L^{2}\left(X^{*}\right)$, while because of hypothesis $H(F)(4)$, and by passing to a subsequence if necessary, we may assume that $f_{n_{k}} \stackrel{w}{\rightarrow} f$ in $L^{2}(H)$. Then for every $v \in L^{2}(H)$ we have

$$
\left(f_{n_{k}}, v\right)_{L^{2}(H)} \leqq \sigma_{\left.S F_{(\cdot,}, x_{m}(\cdot), i_{m}\right)}(v)=\int_{0}^{r} \sigma_{F\left(t, x_{n_{k}}(t), i_{n_{k}}\right)}(v(t)) d t
$$

(see the proof of Theorem 4.2 in Papageorgiou [9]). Also

$$
\begin{aligned}
& \mid \int_{0}^{r}\left(\sigma_{F\left(t, x_{n_{k}}(t), \lambda_{n_{k}}(v(t))-\sigma_{F(t, x(t), \lambda)}(v(t))\right) d t \mid}\right. \\
& \quad \leqq \int_{0}^{r}\left|\sigma_{F\left(t, x_{n_{k}}(t), \lambda_{n_{k}}\right)}(v(t))-\sigma_{F\left(t, x(t), \lambda_{n_{k}}\right)}(v(t))\right| d t+\int_{0}^{r}\left|\sigma_{F\left(t, x(t), \lambda_{n_{k}}\right)}(v(t))-\sigma_{F(t, x(t), \lambda)}(v(t))\right| d t \\
& \quad \leqq \int_{0}^{r} k_{B},(t)\left|x_{n_{k}}(t)-x(t)\right| \cdot|v(t)| d t+\int_{0}^{r} h\left(F\left(t, x(t), \lambda_{n_{k}}\right), F(t, x(t), \lambda)\right)|v(t)| d t \rightarrow 0 .
\end{aligned}
$$

So in the limit we have 


$$
\begin{gathered}
(f, v)_{L^{2}(H)} \leqq \int_{0}^{r} \sigma_{F(t, x(t), \lambda)}(v(t)) d t=\sigma_{\left.S_{F(}^{2}, x(\cdot), i\right)}(v) \quad v \in L^{2}(H) \\
\Rightarrow f \in S_{F(\cdot, x(\cdot), \lambda)}^{2}
\end{gathered}
$$

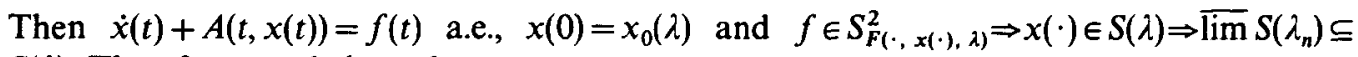
$S(\lambda)$. Therefore we deduce that

$$
S(\cdot) \text { is u.s.c. }
$$

From (1) and (2) above we conclude that $S(\cdot)$ is continuous (for the Vietoris topology on $P_{k}(C(T, H))$ ). But from Corollary 4.2.3, p. 41 of Klein and Thompson [4] we know that on $P_{k}(C(T, H))$ the Vietoris and Hausdorff topologies coincide. So $S(\cdot)$ is also $h$-continuous.

Now we allow operator $A(t, x)$ to depend on the parameter $\lambda$. We will assume a quite general $\lambda$-dependence and for that we need to assume that $A(t, \cdot)$ is linear.

So the multivalued Cauchy problem is now the following

$$
\left\{\begin{array}{l}
\dot{x}(t)+A(t, \lambda) x(t) \in F(t, x(t), \lambda) \\
x(0)=x_{0}(\lambda)
\end{array}\right\}
$$

We will need the following hypothesis on $A(t, \lambda) x$.

$H(A)_{1}: \quad A: T \times \Lambda \rightarrow \mathscr{L}\left(X, X^{*}\right)$ is an operator s.t.

(1) $t \rightarrow A(t, \lambda) x$ is measurable for every $x \in X$,

(2) $\|A(t, \lambda) x\|_{*} \leqq c(\lambda)\|x\|$ a.e. with $c(\lambda)>0$ and $c(\cdot)$ is bounded on compact sets,

(3) $\langle A(t, \lambda) x, x\rangle \geqq c_{1}(\lambda)\|x\|^{2}$ a.e. with $c_{1}(\lambda)>0$ and $1 / c_{1}(\cdot)$ is bounded on compact sets,

(4) if $\lambda_{n} \rightarrow \lambda$ in $\Lambda$, then $A\left(t, \lambda_{n}\right) \stackrel{G}{\rightarrow} A(t, \lambda)$ and given $\varepsilon>0$ there exists

$$
\delta(\varepsilon)>0 \text { s.t. } \sup _{0 \leqq t \leqq r}\left\|A\left(t+\tau, \lambda_{n}\right)-A\left(t, \lambda_{n}\right)\right\|<\varepsilon \quad \text { for } 0<\tau<\delta .
$$

Theorem 3.3. If hypotheses $H(A)_{1}, H(F)_{1}$ and $H_{0}$ hold

$$
\text { then } S: \Lambda \rightarrow P_{k}(C(T, H)) \text { is u.s.c. }
$$

Proof. According to Lemma $\beta$, we need to show that if $\lambda_{n} \rightarrow \lambda$ in $\Lambda$, then $\varlimsup S\left(\lambda_{n}\right) \subseteq S(\lambda)$.

So let $x(\cdot) \in \varlimsup \lim S\left(\lambda_{n}\right)$. By definition we can find $x_{n_{k}} \in S\left(\lambda_{n_{k}}\right), n_{1}<n_{2}<\cdots<n_{k}<\cdots$ s.t. $x_{n_{k}} \stackrel{s}{\rightarrow} x$ in $C(T, H)$. For every $k \geqq 1$, we have 


$$
\left\{\begin{array}{l}
{ }^{*} \dot{x}_{n_{k}}(t)+A\left(t, \lambda_{n_{k}}\right) x_{n_{k}}(t)=h_{n_{k}}(t) \text { a.e. } \\
x_{n_{k}}(0)=x_{0}\left(\lambda_{n_{k}}\right)
\end{array}\right\}
$$

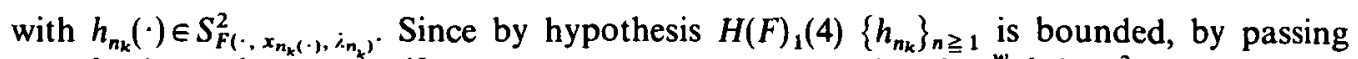
to a further subsequence if necessary, we may assume that $h_{n_{k}} \stackrel{w}{\rightarrow} h$ in $L^{2}(H)$. As in the proof of Theorem 3.2, using the support function, we can show that $h \in S_{F(\cdot, x(\cdot), 3)}^{2}$.

Next fix $y \in W_{r}(T)==\{z \in W(T): z(r)=0\}$ and set

$$
g(t)=-\dot{y}(t)+A(t, \lambda)^{*} y(t) \text { a.e. }
$$

Let $\left\{y_{n_{k}}\right\}_{k \geqq 1} \subseteq W(T)$ be the solutions of the Cauchy problems

$$
-\dot{y}_{n_{k}}(t)+A\left(t, \lambda_{n_{k}}\right)^{*} y_{n_{k}}(t)=g(t) \text { a.e., } y_{n_{k}}(r)=0 .
$$

From Theorem 7 of Zhikov, Kozlov and Oleinik [16] we know that

$$
P_{n_{k}}=\frac{d}{d t}+\hat{A}\left(\lambda_{n_{k}}\right) \stackrel{P G}{\rightarrow} P=\frac{d}{d t}+\hat{A}(\lambda)
$$

So

$$
P_{n_{k}}^{*}=-\frac{d}{d t}+\hat{A}\left(\lambda_{n_{k}}\right)^{*} \stackrel{P G}{\rightarrow} P^{*}=-\frac{d}{d t}+\hat{A}(\lambda)^{*}
$$

(Theorem 10 of Zhikov, Kozlov and Oleinik [16]). Hence invoking Lemma 3 of [16], we have that $y_{n_{k}} \stackrel{s}{\rightarrow} y$ in $C(T, H)$.

Multiply equation (1) with $y_{n_{k}}(\cdot)$, equation (2) with $x_{n_{k}}(\cdot)$ and then subtract the new equation (2) from the new equation (1) and integrate over $T$. We get

$$
\begin{aligned}
\int_{0}^{r}\left\langle\dot{x}_{n_{k}}(t), y_{n_{k}}(t)\right\rangle d t & \left.+\int_{0}^{r}\left\langle\dot{y}_{n_{k}}(t), x_{n_{k}}(t)\right\rangle d t+\int_{0}^{r}\left\langle A\left(t, \lambda_{n_{k}}\right) x_{n_{k}}\right)(t), y_{n_{k}}(t)\right\rangle d t \\
& -\int_{0}^{r}\left\langle A\left(t, \lambda_{n_{k}}\right)^{*} y_{n_{k}}(t), x_{n_{k}}(t)\right\rangle d t \\
& =\int_{0}^{r}\left\langle h_{n_{k}}(t), y_{n_{k}}(t)\right\rangle d t-\int_{0}^{r}\left\langle g(t), x_{n_{k}}(t)\right\rangle d t .
\end{aligned}
$$

Using Lemma 5.5 .1 of Tanabe [12], we can perform integration by parts on the second integrand of the left-hand side and get

$$
\int_{0}^{r}\left\langle\dot{y}_{n_{k}}(t), x_{n_{k}}(t)\right\rangle d t=\left(y_{n_{k}}(r), x_{n_{k}}(r)\right)-\left(y_{n_{k}}(0), x_{n_{k}}(0)\right)-\int_{0}^{r}\left\langle y_{n_{k}}(t), \dot{x}_{n_{k}}(t)\right\rangle d t
$$




$$
=-\left(y_{n_{k}}(0), x\left(0, \lambda_{n_{k}}\right)\right)-\int_{0}^{r}\left\langle y_{n_{k}}(t), \dot{x}_{n_{k}}(t)\right\rangle d t
$$

Substituting (4) in (3) we get

$$
\begin{aligned}
&-\left(y_{n_{k}}(0), x\left(0, \lambda_{n_{k}}\right)\right)=\int_{0}^{r}\left\langle h_{n_{k}}(t), y_{n_{k}}(t)\right\rangle d t-\int_{0}^{r}\left\langle g(t), x_{n_{k}}(t)\right\rangle d t-(y(0), x(0, \lambda))=\int_{0}^{r}\langle h(t), y(t)\rangle d t-\int_{0}^{r}\langle g(t), x(t)\rangle d t \\
& \Rightarrow \int_{0}^{r}\langle h(t), y(t)\rangle d t-\int_{0}^{r}\left\langle-\dot{y}(t)+A(t, \lambda)^{*} y(t), x(t)\right\rangle d t \\
&= \int_{0}^{r}\langle h(t), y(t)\rangle d t-(y(0), x(0, \lambda))-\int_{0}^{r}\langle y(t), \dot{x}(t)\rangle d t \\
&-\int_{0}^{r}\langle y(t), A(t, \lambda) x(t)\rangle d t \\
& \Rightarrow \int_{0}^{r}\langle\dot{x}(t)+A(t, \lambda) x(t), y(t)\rangle d t=\int_{0}^{r}\langle h(t), y(t)\rangle d t .
\end{aligned}
$$

Since $y(\cdot) \in W_{r}(T)$ was arbitrary and $W_{r}(T)$ is dense in $L^{2}(X)$, we conclude that

$$
\begin{aligned}
& \dot{x}(t)+A(t, \lambda) x(t)=h(t) \text { a.e., } x(0)=x_{0}(\lambda), h \in S_{F(\cdot, x(\cdot), \lambda)}^{2} \\
& \Rightarrow \quad x(\cdot) \in S(\lambda) \\
& \Rightarrow \quad \varlimsup S\left(\lambda_{n}\right) \subseteq S(\lambda) \\
& \Rightarrow S(\cdot) \text { is u.s.c. }
\end{aligned}
$$

\section{Applications}

In this section we present two examples illustrating the applicability of our work.

The first example is from control theory. So let $Z$ be a bounded domain in $\mathbb{R}^{n}$ with smooth boundary $\partial Z=\Gamma$. We consider the following nonlinear parabolic distributed parameter control system, in divergence form: 


$$
\left\{\begin{array}{l}
\frac{\partial x}{\partial t}+\sum_{|\alpha| \leqq m}(-1)^{|\alpha|} D_{\alpha} A_{\alpha}(t, z, \eta(x(z)))=f(t, z, x(t, z), \lambda) u(t, z) \text { on } T \times Z \\
D^{\beta} x(t, z)=0 \text { on } T \times \Gamma \text { for }|\beta| \leqq m-1 \\
\quad x(0, z)=x_{0}(z, \lambda) \\
\int_{Z}|u(t, z)|^{2} d z \leqq r(t, \lambda)^{2} \text { a.e. } \text { with } u(\cdot, \cdot) \text { measurable }
\end{array}\right\}
$$

Here $\eta(x(z))=\left\{D^{\alpha} x(z):|\alpha| \leqq m\right\}$ (i.e. the tuple of all partial derivatives up to order $m$ including $x(\cdot))$.

We denote the set of trajectories of $(* * *)$ by $S(\lambda) \subseteq C\left(T, L^{2}(Z)\right)$.

We will need the following hypotheses on the data of $\left({ }^{* *}\right)_{1}$.

$$
H(A)_{2}: \quad A_{\alpha}: T \times Z \times \mathbb{R}^{n(m)} \rightarrow \mathbb{R}\left(n(m)=\frac{(n+m) !}{n ! m !}\right) \text { are maps s.t. }
$$

(1) $(t, z) \rightarrow A_{\alpha}(t, z, \eta)$ are measurable,

(2) $\eta \rightarrow A_{\alpha}(t, z, \eta)$ are continuous,

(3) $\left|A_{\alpha}(t, z, \eta)\right| \leqq \phi(t, z)+c\|\eta\|$ a.e. with $\phi(\cdot, \cdot) \in L_{2}(T \times Z), c>0$,

(4) $\sum_{|\alpha| \leqq m}\left(A_{\alpha}\left(t, z, \eta_{1}\right)-A\left(t, z, \eta_{2}\right)\right)\left(\eta_{1 \alpha}-\eta_{2 \alpha}\right) \geqq 0$,

(5) $\sum_{|\alpha| \leqq m} A_{\alpha}(t, z, \eta) \eta_{\alpha} \geqq c_{0}\|\eta\|^{2} c_{0}>0$.

$H(f): \quad f: T \times Z \times \mathbb{R} \times \Lambda \rightarrow \mathbb{R}$ is a map s.t.

(1) $(t, z) \rightarrow f(t, x, z, \lambda)$ is measurable,

(2) $|f(t, z, x, \lambda)-f(t, z, y, \lambda)| \leqq k_{B}(t, z)|x-y|$ a.e. for all $\lambda \in B \leqq \Lambda$ compact and $k_{B}(\cdot, \cdot) \in L_{+}^{\infty}$,

(3) $\lambda \rightarrow f(t, z, x, \lambda)$ is continuous,

(4) $|f(t, z, x, \lambda)| \leqq a_{B}(t, z)+b_{B}(z)|x|$ a.e. for all $\lambda \in B \subseteq \Lambda$ compact and with $a_{B}(\cdot, \cdot) \in L_{+}^{2}(T \times Z) b_{B}(\cdot) \in L_{+}^{2}(Z)$.

$H(r): \quad r(\cdot, \lambda) \in L_{+}^{\infty}, r(t, \cdot)$ is continuous and $\sup _{\lambda \in B}\|r(\cdot, \lambda)\|_{\infty}=\hat{M}_{B}<\infty$ for all $B \subseteq \Lambda$ compact.

$H_{0}: \quad x_{0}(\cdot, \lambda) \in L^{2}(Z)$ and $\lambda \rightarrow x_{0}(\cdot, \lambda)$ is continuous from $\Lambda$ into $L^{2}(Z)$

In this case $X=H_{0}^{m}(Z), H=L^{2}(Z)$ and $X^{*}=H^{-m}(Z)$. Then $\left(X, H, X^{*}\right)$ is a Gelfand triple of spaces with all embedding being compact (Sobolev-Kondrachov embedding theorem).

Consider the time varying Dirichlet formula $a: T \times H_{0}^{m}(Z) \times H_{0}^{m}(Z) \rightarrow \mathbb{R}$ corresponding to the elliptic operator of our system and defined by 


$$
a(t, x, y)=\sum_{|\alpha| \leqq m} \int_{Z} A_{\alpha}(t, z, \eta(x(z))) D^{\alpha} y(z) d z, \quad x, y \in H_{o}^{m}(Z) .
$$

A simple argument involving the Cauchy-Schwartz and Hölder inequalities tells us that

$$
\left.|a(t, x, y)| \leqq 2 \cdot[\| \phi t, \cdot)\left\|_{L^{2}(Z)}+\hat{c}\right\| x \|_{H_{0}^{m}(Z)}\right] \cdot\|y\|_{H_{0}^{m}(Z)}
$$

with $\hat{c}>0$. So we can find a generally nonlinear operator $A(t, \cdot): H_{0}^{m}(Z) \rightarrow H^{-m}(Z)$ s.t.

$$
a(t, x, y)=\langle A(t, x), y\rangle \text {. }
$$

Clearly by Fubini's theorem $t \rightarrow\langle A(t, x), y\rangle$ is measurable $\Rightarrow A(\cdot, x)$ weakly measurable and since $X^{*}$ is separable, by Pettis' theorem, we conclude that $t \rightarrow A(t, x)$ is measurable. Also for every $x_{n} \stackrel{s}{\rightarrow} x$ in $H_{0}^{m}(Z)$, we have

$$
\left|\left\langle A\left(t, x_{n}\right)-A(t, x), y\right\rangle\right| \leqq \sum_{|\alpha| \leqq m}\left|\int_{Z}\left(A_{\alpha}\left(t, z, \eta\left(x_{n}(z)\right)\right)-A_{\alpha}(t, z, \eta(x(z)))\right) D^{\alpha} y(z) d z\right| \rightarrow 0
$$

because of hypotheses $H(A)_{1}(1),(2)$ and (3). Thus $A(t, \cdot)$ is demicontinuous, hence hemicontinuous too.

Also it is easy to see that because of $H(A)_{1}(4), A(t, \cdot)$ is monotone, while from $H(A)_{1}(5)$ we have that $\langle A(t, x), x\rangle \geqq \hat{c}_{0}\|x\|_{H_{0}^{m}(Z)}^{2}$ with $\hat{c}>0$. So we have satisfied hypothesis $H(A)$.

Next let $\hat{f}: T \times L^{2}(Z) \times \Lambda \rightarrow L^{2}(Z)$ be defined by

$$
\hat{f}(t, x, \lambda)(z)=f(t, z, x(z), \lambda)
$$

i.e., $\hat{f}(\cdot, \cdot, \cdot)$ is the Nemitsky (superposition) operator corresponding to $f$. Then for every $y \in L^{2}(Z)$, we have $(\hat{f}(t, x, \lambda), y)_{L^{2}(Z)}=\int_{Z} f(t, z, x(z), \lambda) y(z) d z \Rightarrow t \rightarrow(\hat{f}(t, x, \lambda), y)_{L^{2}(Z)}$ is measurable (Fubini's theorem) $\Rightarrow \hat{f}(\cdot, x, \lambda)$ is weakly measurable and since $L^{2}(Z)$ is separable, again by Pettis' theorem, we conclude that $\hat{f}(\cdot, x, \lambda)$ is measurable.

Also

$$
\begin{aligned}
\|\hat{f}(t, x, \lambda)-\hat{f}(t, y, \lambda)\|_{L^{2}(Z)}^{2} & =\int_{Z}|f(t, z, x(z), \lambda)-f(t, z, y(z), \lambda)|^{2} d z \\
& \leqq \int_{Z} k_{B}(t, z)^{2}|x(z)-y(z)|^{2} d z \\
& \leqq\left\|k_{B}(t, \cdot)\right\|_{L^{\infty}(Z)}^{2}\|x-y\|_{L^{2}(Z)} \text { a.e. }
\end{aligned}
$$

$\Rightarrow \hat{f}(t, \cdot, \lambda)$ is Lipschitz continuous. Furthermore through the dominated convergence theorem we can check that $\hat{f}(t, x, \cdot)$ is continuous. 
Next let $U: T \times \Lambda \rightarrow P_{f c}\left(L^{2}(Z)\right)$ be defined by

$$
U(t, \lambda)=\left\{u \in L^{2}(Z):\|u\|_{L^{2}(Z)} \leqq r(t, \lambda)\right\} .
$$

Then because of hypothesis $H(r)$ it is easy to check that $U(\cdot, \lambda)$ is measurable, $U(t, \cdot)$ is continuous and $|U(t, \lambda)| \leqq\|r(\cdot, \lambda)\|_{\infty}$.

Now let $F: T \times L^{2}(Z) \times \Lambda \rightarrow P_{f c}\left(L^{2}(Z)\right)$ be defined by

$$
\hat{F}(t, x, \lambda)=\hat{f}(t, x, \lambda) U(t, \lambda) .
$$

Then

$h(\hat{F}(t, x, \lambda), \hat{F}(t, y, \lambda)) \leqq\|\hat{f}(t, x, \lambda)-\hat{f}(t, y, \lambda)\|_{L^{2}(Z)}\|r(\cdot, \lambda)\|_{\infty} \leqq\left\|k_{B}(t, \cdot)\right\|_{L^{\infty}(Z)}\|x-y\|_{L^{2}(Z)} \hat{M}_{B}$.

Also if $\lambda_{n} \rightarrow \lambda$ in $\Lambda$ we have

$$
\begin{aligned}
h\left(\hat{F}\left(t, x, \lambda_{n}\right), \hat{F}(t, y, \lambda)\right) \leqq & h\left(\hat{f}\left(t, x, \lambda_{n}\right) U\left(t, \lambda_{n}\right), \hat{f}(t, x, \lambda) U\left(t, \lambda_{n}\right)\right) \\
& +h\left(\hat{f}(t, x, \lambda) U\left(t, \lambda_{n}\right), \hat{f}(t, x, \lambda) U(t, \lambda)\right) \\
\leqq & \left\|\hat{f}\left(t, x, \lambda_{n}\right)-\hat{f}(t, x, \lambda)\right\|_{L^{2}(Z)} \cdot \hat{M}_{B}\left(\text { here } B=\left\{\lambda_{n}, \lambda\right\}_{n \geqq 1} \subseteq \Lambda\right) \\
& +\|\hat{f}(t, x, \lambda)\|_{L^{2}(Z)} h\left(U\left(t, \lambda_{n}\right), U(t, \lambda)\right) \rightarrow 0 .
\end{aligned}
$$

$\Rightarrow \hat{F}(t, x, \cdot)$ is $h$-continuous. So we have satisfied hypothesis $H(F)_{1}$.

Then consider the following evolution inclusion:

$$
\left\{\begin{array}{l}
\dot{x}(t)+A(t, x(t)) \in \hat{F}(t, x(t), \lambda) \text { a.e. } \\
x(0)=\hat{x}_{0}(\lambda)=x_{0}(\cdot, \lambda)
\end{array}\right\}
$$

A straightforward argument involving Aumann's selection theorem shows that $\left({ }^{* * *}\right)_{1}$, is equivalent to the following abstract control problem

$$
\left\{\begin{array}{ll}
\dot{x}(t)+A(t, x(t))=\hat{f}(t, x(t), \lambda) u(t) \text { a.e. } \\
x(0)=\hat{x}_{0}(\lambda), & u(t) \in U(t, \lambda) \text { a.e. } \\
& u(\cdot) \text { is measurable }
\end{array}\right\}
$$

From all the previous work, we know that $\left({ }^{* *}\right)_{1}$, is equivalent to $\left({ }^{* *}\right)_{1}$.

Note the problem $\left({ }^{* * *}\right)_{1}$, has the form of $\left({ }^{*}\right)_{\lambda}$ and we saw it is equivalent to $\left({ }^{* *}\right)_{1}$. So invoking Theorem 3.2 we get:

Theorem 4.1. If hypotheses $H(A)_{2} . H(f), H(r)$ and $H_{0}$ hold, then $S: \Lambda \rightarrow P_{k}\left(C\left(T, L^{2}(Z)\right)\right)$ is continuous and h-continuous. 
Also let $R(t, \lambda)=\left\{x(t, \cdot) \in L^{2}(Z): x \in S(\lambda)\right\}$ be the reachable set at time $t \in T$. Then, since $R(t, \lambda)=e_{t}(S(\lambda))$ where $e_{t}$ is the evaluation map at $t \in T$, we have:

Theorem 4.2. If hypotheses $H(A)_{2}, H(f), H(r)$ and $H_{0}$ hold, then $R(t, \cdot): \Lambda \rightarrow P_{k}\left(L^{2}(Z)\right)$ is continuous and h-continuous.

Our second example is from parabolic partial differential inclusions. Such inclusions arise in the study of obstacle problems and of partial differential equations with discontinuous noninearities (see Chang [2]). Here let $Z=[0,1]$ (i.e. $n=1$ ).

So we consider the following initial-boundary value problem on $T \times Z=[0, b] \times[0,1]$ :

$$
\left\{\begin{array}{l}
\frac{\partial x}{\partial t}-\frac{\partial}{\partial z}(u(z, \lambda)) \frac{\partial x}{\partial z} \in F(t, z, x(t, x), \lambda) \quad \text { on } T \times X \\
\left.x\right|_{T \times \Gamma}=0, \quad x(0, z)=x_{0}(z, \lambda)
\end{array}\right\}
$$

Denote its solution set by $S(\lambda) \subseteq C\left(T, L^{2}(Z)\right)$.

We will need the following hypotheses on the data of $(* * *)_{2}$.

$H(A)_{3}: \quad 0<m_{1 B} \leqq u(z, \lambda) \leqq m_{2 B}$ for $\lambda \in B \subseteq \Lambda$ compact and if $\lambda_{n} \rightarrow \lambda$, then

$$
\frac{1}{u\left(\cdot, \lambda_{n}\right)} \stackrel{w^{*}}{\rightarrow} \frac{1}{u(\cdot, \lambda)} \text { in } L_{+}^{\infty}(Z)
$$

$H(F)_{2}: \quad F(t, z, x, \lambda)=\left[v_{1}(t, z, x, \lambda), v_{2}(t, z, x, \lambda)\right]$, where $v_{i}: T \times Z \times \mathbb{R} \times \Lambda \rightarrow \mathbb{R} \quad i=1,2$ are maps s.t. $v_{1} \leqq v_{2}$

(1) $(t, z) \rightarrow v_{i}(t, z, x, \lambda)$ are measurable,

(2) $\left|v_{i}(t, z, x, \lambda)-v_{i}(t, z, y, \lambda)\right| \leqq k_{B}(t, z)|x-y|$ a.e. for all $\lambda \in B \subseteq \Lambda$ compact and $k_{B}(\cdot, \cdot) \in L^{\infty}(T \times Z)$,

(3) $\left|v_{i}(t, z, x, \lambda)\right| \leqq a_{B}(t, z)+b_{B}(z)|x|$ a.e. for all $\lambda \in B \subseteq \Lambda$ compact and with $a_{B}(\cdot, \cdot) \in L_{+}^{2} b_{B}(\cdot) \in L_{+}^{\infty}$.

Here the Gelfand triple is $X=H_{0}^{1}(Z), H=L^{2}(Z)$ and $X^{*}=H^{-1}(Z)$. All embeddings are compact.

As in the first example, through the Dirichlet form corresponding to the elliptic operator of our problem, we can define a map $A: \Lambda \rightarrow \mathscr{L}\left(H_{0}^{1}(Z), H^{-1}(Z)\right)$ s.t.

$$
\langle A(\lambda) x, y\rangle=\int_{z} u(z, \lambda) \frac{\partial x}{\partial z} \frac{\partial y}{\partial z} d z
$$

Because of hypothesis $H(A)_{3}$ and using Tartar's theorem (see Sokolowski [10, Lemma 3 , p. 289]), we have that if $\lambda_{n} \rightarrow \lambda$ in $\Lambda$, then $A\left(\lambda_{n}\right) \stackrel{G}{\rightarrow} A(\lambda)$. 
Also let $\hat{F}: T \times L^{2}(Z) \times \Lambda \rightarrow P_{f c}\left(L^{2}(Z)\right)$ be defined by

$$
\begin{aligned}
\hat{F}(t, x, \lambda) & =\left[\hat{v}_{1}(t, x, \lambda)(\cdot), \hat{v}_{2}(t, x, \lambda)(\cdot)\right]=\left\{w \in L^{2}(Z): \hat{v}_{1}(t, x, \lambda)(z) \leqq w(z)\right. \\
& \left.\leqq \hat{v}_{2}(t, x, \lambda)(z) \text { a.e. }\right\}
\end{aligned}
$$

where $\hat{v}_{i} i=1,2$ are the Nemitsky operators corresponding to the functions $v_{i} i=1,2$. Using hypothesis $H(F)_{2}$ it is easy to check that $\hat{F}(t, x, \lambda)$ defined as above satisfies $H(F)_{1}$.

Then $\left({ }^{* *}\right)_{2}$ is equivalent to the following evolution inclusion:

$$
\left\{\begin{array}{l}
\dot{x}(t)+A(\lambda) x(t) \in \hat{F}(t, x(t), \lambda) \text { a.e. } \\
x(0)=\hat{x}_{0}(\lambda)=x_{0}(\cdot, \lambda)
\end{array}\right\} .
$$

Invoking Theorem 3.3 we have:

Theorem 4.3. If hypotheses $H(A)_{3}, H(F)_{2}$ and $H_{0}$ hold, then $S: \Lambda \rightarrow P_{k}\left(C\left(T, L^{2}(Z)\right)\right)$ is u.s.c.

Acknowledgement. The author would like to thank the referee for his corrections and detailed and knowledgeable comments.

\section{REFERENCES}

1. V. Barbu, Nonlinear Semigroups and Differential Equations in Banach Spaces (Noordhoff International Publishing, Leyden, The Netherlands, 1976).

2. K. C. Chang, The obstacle problem and partial differential equations with discontinuous nonlinearities, Comm. Pure Appl. Math. 33 (1980), 117-146.

3. J. Delahaye and J. Denel, The continuities of the point to set maps, definitions and equivalences, Math. Programming Study 10 (1979), 8-12.

4. E. Klein and A. Thompson, Theory of Correspondences (Wiley-Interscience, New York, 1984).

5. T.-C. Lim, On fixed point stability for set valued contractive mappings with applications to generalized differential equations, J. Math. Anal. Appl. 110 (1985), 436-441.

6. J.-L. Lıons, Quelques Méthodes de Résolution des Problèmes aux Limites Non Linéaires (Dunod-Gauthier Villars, Paris, 1969).

7. E. NAGY, A theorem on compact embedding for functions with values in an infinite dimensional Hilbert space, Annales Univ. Sci. Budapest 23 (1980), 243-245.

8. N. S. Papageorgiou, A stability result for differential inclusions in Banach spaces, J. Math. Anal. Appl. 118 (1986), 232-246.

9. N. S. Papageorgiou, Convergence theorems for Banach space valued integrable multifunctions, Internat. J. Math. Math. Sci. 10 (1987), 443-442.

10. J. Sokolowskı, Optimal control in coefficients for weak varitional problems in Hilbert space, Appl. Math. Optim. 7 (1981), 283-293. 
11. G. Stassinopoulos and R. Vinter, Continuous dependence of solutions of a differential inclusion on the right hand side with applications to stability of optimal control problems, SIAM J. Control Optim. 17 (1979), 432-449.

12. H. Tanabe, Equations of Evolution (Pitman, London, 1979).

13. A. Tolstonogov, On the dependence on a parameter of a solution of a differential inclusion with a nonconvex second member, Differential Equations 18 (1982), 1105-1113.

14. A. B. VASILEv, Continuous dependence of the solutions of differential inclusions on the parameter, Ukranian Math. J. 35 (1983), 520-524.

15. D. WAGNER, Survey of measurable selection theorems, SIAM J. Control Optim. 15 (1977), 859-903.

16. V. Zhikov, S. Kozlov and O. Oleinik, G-convergence of parabolic operators, Russian Math. Surveys 36 (1981), 9-60.

17. H. FrankowSKa, A priori estimates for operational differential inclusions, J. Differential Equations 84 (1990), 100-128.

18. N. S. Papageorgiou, Relaxation and existence of optimal controls for systems governed by evolution inclusions in separable Banach spaces, J. Optim. Theory Appl. 64 (1990), 573-594.

19. E. ZeIdLer, Nonlinear Functional Analysis and its Applications, Vol. II (Springer, Berlin, 1990).

20. S. Spagnolo, Sul limite delle soluzioni di problemi di Cauchy relativi all' equazioni del calore, Ann. Scuola Norm. Sup. Pisa 21 (1967), 657-699.

21. S. Spagnolo, Convergence of parabolic operators, Boll. Un. Mat. Ital. 14 (1977), 547-568.

22. F. Colombins and S. Spagnolo, Sur la convergence des solutions des équations paraboliques, J. Math. Pures Appl. 56 (1977), 263-306.

National Technical University

Department of Mathematics

Athens 15773, Greece 